\title{
Bone mineral density changes in pregnancies with gestational hypertension: a longitudinal study using quantitative ultrasound measurements
}

\author{
William W. K. To $\cdot$ Margaret W. N. Wong
}

Received: 18 May 2010 / Accepted: 6 July 2010 / Published online: 22 July 2010

(C) The Author(s) 2010. This article is published with open access at Springerlink.com

\begin{abstract}
Objective To compare the maternal bone mineral density (BMD) changes in gestational hypertensive and normotensive pregnancies using quantitative ultrasound.

Methods Consecutive patients were recruited from a general obstetric clinic over a period of 9 months. BMD measurements were performed at the os calcis in early pregnancy before 20 weeks and in the late third trimester after 36 weeks, using a Hologic Sahara Clinical Bone Sonometer system. These patients were followed up in accordance with standard antenatal protocol. The diagnosis of gestational hypertension (GH) was made based on a standard institutional protocol. The changes in BMD from early to late pregnancy were compared between those with/ without GH.

Results A total of 450 patients with complete data were analyzed. The overall incidence of GH was $4.8 \%(n=22)$, of which $1.7 \%(n=8)$ fulfilled the definitions of severe preeclampsia. A mean BMD loss of $0.0256 \mathrm{~g} / \mathrm{cm}^{2}$ (around $4.5 \%$ of early pregnancy BMD) was demonstrable from early to late gestation. The hypertensive group has marginally higher mean BMD loss as compared to the normoten-
\end{abstract}

W. W. K. To $(\bowtie)$

Department of Obstetrics and Gynaecology, United Christian Hospital, 130 Hip Wo Street,

Kwun Tong, Kowloon, Hong Kong

e-mail: towkw@ha.org.hk

M. W. N. Wong

Department of Orthopaedics and Traumatology,

Prince of Wales Hospital, Shatin, Hong Kong sive group ( 0.052 vs. $\left.0.037 \mathrm{~g} / \mathrm{cm}^{2} ; P=0.037\right)$. However, regression analysis models showed that early pregnancy BMD values, early pregnancy fat percentage and fat accumulation in pregnancy were significant factors affecting BMD loss during pregnancy, while GH was not in the equations.

Conclusion The development of gestational hypertensive disorders apparently does not have any significant impact on BMD changes during pregnancy.

Keywords Bone mineral density - Gestational hypertension - Quantitative ultrasound

\section{Introduction}

Studies utilizing different methods of assessment, including radiological [1,2] and quantitative ultrasound [3, 4], have been used to study the changes in bone mineral density (BMD) during pregnancy, and a progressive fall in BMD is usually demonstrated from the pre-pregnant state to early postpartum, or from early pregnancy to late pregnancy. While the factors that would influence the magnitude of BMD changes in pregnancy have been studied in various settings, the actual patho-physiology involved in such control is still largely unknown. In particular, few studies have addressed how particular antenatal complications would affect such changes. Gestational hypertension $(\mathrm{GH})$ and pre-eclampsia are common obstetric problems that would develop usually during the latter part of the pregnancy. This study aims at evaluating the differences in BMD changes of the os calcis as measured by quantitative ultrasound from early to late pregnancy in women with gestational hypertensive disorders, in order to verify whether such conditions would have an impact on BMD changes in pregnancy. 


\section{Methods}

A prospective observational study was conducted in a regional obstetric unit with an annual delivery of around 5,000 deliveries. Consecutive patients with singleton booked at a general obstetric clinic were recruited over a 9-month period. Routine antenatal care was offered in accordance with our service protocol. Written consent for quantitative ultrasound BMD measurements of the os calcis was obtained at the time of recruitment. Basic epidemiological data, including early pregnancy weight and height, were recorded. Quantitative ultrasound bone density measurements were performed at the os calcis bilaterally at booking between 14 and 20 weeks, and in the third trimester between 36 and 38 weeks. All bone density measurements were done using the Sahara Clinical Bone Sonometer system (Hologic, MA, USA), a waterless portable system that involved direct contact of the probe with the heel through elastomer pads and oil based coupling gel. A simulated BMD value derived from basic speed of sound and bone ultrasound attenuation parameters was obtained, and this was used in the subsequent analysis for calculations. The coefficient of variation for the estimated heel BMD value was estimated to be around $2-3 \%$ based on the authors' previous studies utilizing the same system. Body fat percentage assay was also performed in each of the two occasions using a Tanita 500 bio-impedance system (Tanita, Tokyo, Japan). The excitation current introduced into subjects was $800 \mu \mathrm{A}, \mathrm{AC}$ at $50 \mathrm{kHz}$. The analyzer enabled simultaneous measurements of body weight and impedance in a subject standing on the stainless steel electrodes with their bare feet, and calculated the percentage of body fat based on a two-compartment model of fat mass and lean body mass. The coefficient of variation of the system was quoted as $2.5-3.5 \%$ according to the manufacturer. Patients with other confounding significant medical disorders during the antenatal period, including pre-existing medical disorders requiring treatment such as asthma or thyroid disorders, and those that delivered preterm before 37 weeks gestation, or who did not complete the second measurement were excluded from analysis.

Blood pressure was monitored at all regular antenatal visits, and GH was diagnosed when the pregnant woman had blood pressure over $140 / 90 \mathrm{mmHg}$ for two episodes or more, $6 \mathrm{~h}$ apart at gestation 20 weeks or beyond. Preeclampsia was diagnosed when significant proteinuria of over $300 \mathrm{mg}$ was quantitatively documented in a 24-h urine sample. Eclampsia referred to patients with the overt clinical observation of an eclamptic fit superimposed on preeclampsia, and cases with subtle peripheral twitching or electroencephalographic signs only were excluded. Chronic hypertension was diagnosed when the woman was documented to have a blood pressure of over $140 / 90 \mathrm{mmHg}$ before 20 weeks or who was diagnosed as hypertensive before the index pregnancy. Women diagnosed with chronic hypertension were excluded from the final analysis, unless there was definite clinical evidence of superimposed pre-eclampsia. The criteria used in the study were adapted from the local College guidelines and are similar to those of established consensus standards [5-7].

The occurrence of GH or pre-eclampsia was correlated with the degree of os calcis BMD changes from early to late gestation. Other possible confounding factors that might affect BMD changes in pregnancy were then analyzed, including the early pregnancy body mass index, early pregnancy BMD value, and fat accumulation during pregnancy. Univariate analysis of the data was performed by Chi-square tests for discrete entities, and by paired and unpaired Student's $t$ tests for continuous variables where appropriate, with prior testing for normal distribution of the data. Parameters found likely to be statistically significant on univariate analysis were then entered into a logistic regression model, as well as a multiple regression model, with BMD loss in pregnancy as the dependent variable. Data were analyzed using the SPSS version 14.0 (SPSS, Chicago, IL, USA). A $P$ value of $<0.05$ was considered significant in this study. The study was approved of by the Ethics Committee of the local cluster hospital board.

\section{Results}

A total of 450 patients with complete data were analyzed. The overall incidence of GH was $4.8 \%(n=22)$, of which $1.7 \%(n=8)$ fulfilled the definitions of severe preeclampsia. The trends in body weight, body mass index and body fat percentage changes from early to late pregnancy were also highly significant (Table 1 ). A mean BMD loss of $0.0256 \mathrm{~g} / \mathrm{cm}^{2}$ (around $4.5 \%$ of early pregnancy BMD) was demonstrable from early to late gestation (Table 1).

There were no significant differences in the age, education levels or occupation categories between those who developed GH and those without. A higher proportion in the GH group was primiparous ( 82 vs. $47.9 \%, P=0.025$ ). The GH group had significantly higher early pregnancy weight, body mass index, and body fat percentage, as well as a marginally higher early pregnancy BMD value (Table 2). While the mean weight and body fat gain during pregnancy were not different between the two groups, the GH group had a marginally higher BMD loss as compared to the normotensive group $\left(0.052\right.$ vs. $0.037 \mathrm{~g} / \mathrm{cm}^{2}$, $P=0.037)$. Within the hypertensive pregnancies, there were no significant differences in BMD loss between those with GH or pre-eclampsia, probably because of the small numbers involved. 
Table 1 Changes in anthropometric parameters from early to late pregnancy

\begin{tabular}{lccc}
\hline & $\begin{array}{l}\text { Early pregnancy } \\
(<20 \text { weeks })(\mathrm{SD})\end{array}$ & $\begin{array}{l}\text { Late third trimester } \\
(36-40 \text { weeks })(\mathrm{SD})\end{array}$ & $P$ value; MD (95\% CI) \\
\hline Weight $(\mathrm{kg})$ & $56.9(8.37)$ & $66.6(8.11)$ & $<0.001 ; 9.78(9.5$ to 10.07$)$ \\
Body mass index $\left(\mathrm{kg} / \mathrm{cm}^{2}\right)$ & $23.2(3.23)$ & $27.2(3.08)$ & $<0.001 ; 4.0(3.88$ to 4.11$)$ \\
Body fat composition $(\%)$ & $30.5(5.79)$ & $38(4.95)$ & $<0.001 ; 7.52(7.25$ to 7.79$)$ \\
Mean BMD $\left(\mathrm{g} / \mathrm{cm}^{2}\right)$ & $0.593(0.108)$ & $0.567(0.102)$ & $<0.001 ;-0.025(-0.029$ to -0.021$)$ \\
\hline
\end{tabular}

$P$ value by paired $t$ tests

$S D$ standard deviation, $M D$ mean difference, $C I$ confidence interval

Table 2 Mean anthropometric and BMD changes in those with or without gestational hypertension

\begin{tabular}{|c|c|c|c|c|}
\hline & Gestational hypertension $(n=22)$ & Normal $(n=428)$ & $P$ value & $\operatorname{MD}(95 \% \mathrm{CI})$ \\
\hline Age (years) & $31.2(\mathrm{SD} 5.29)$ & $31.1(\mathrm{SD} 4.06)$ & 0.93 & $0.07(-1.69$ to 1.85$)$ \\
\hline Height $(\mathrm{cm})$ & $156.4(\mathrm{SD} 5.11)$ & $156.4(\mathrm{SD} 5.39)$ & 0.97 & $-0.03(-2.35$ to 2.28$)$ \\
\hline \multicolumn{5}{|l|}{ Education } \\
\hline Primary & $0(0 \%)$ & $8(1.9 \%)$ & \multirow[t]{3}{*}{ NS } & \\
\hline Secondary & $18(82 \%)$ & $378(88.3 \%)$ & & \\
\hline Tertiary & $4(18 \%)$ & $42(9.8 \%)$ & & \\
\hline \multicolumn{5}{|l|}{ Parity } \\
\hline Primiparous & $18(81.8 \%)$ & $223(52.1 \%)$ & \multirow[t]{3}{*}{0.025} & \\
\hline Para 1 & $4(18.2 \%)$ & $182(42.5 \%)$ & & \\
\hline Para 2 or higher & 0 & $23(5.4 \%)$ & & \\
\hline \multicolumn{5}{|l|}{ Occupation } \\
\hline Professional & $2(9 \%)$ & $10(2.3 \%)$ & \multirow[t]{6}{*}{ NS } & \\
\hline Clerical & $10(45.5 \%)$ & $144(33.7 \%)$ & & \\
\hline Technical & $4(18.1 \%)$ & $40(9.4 \%)$ & & \\
\hline Service & $3(13.7 \%)$ & $112(26.1 \%)$ & & \\
\hline Manual & $0(0 \%)$ & $44(10.3 \%)$ & & \\
\hline Housewife & $3(13.7 \%)$ & $78(18.2 \%)$ & & \\
\hline Early pregnancy weight (kg) & $61.9(\mathrm{SD} 10.6)$ & $56.6(\mathrm{SD} 8.17)$ & 0.004 & $5.29(1.73$ to 8.86$)$ \\
\hline Early pregnancy BMI $\left(\mathrm{kg} / \mathrm{cm}^{2}\right)$ & $25.3(\mathrm{SD} 4.14)$ & $23.1(\mathrm{SD} 3.14)$ & 0.002 & $2.17(0.80$ to 3.55$)$ \\
\hline Early pregnancy body fat (\%) & 34.7 (SD 6.03) & $30.3(\mathrm{SD} 5.70)$ & 0.001 & 4.47 (2.01 to 6.93$)$ \\
\hline Early pregnancy BMD (g/cm²) & $0.636(\mathrm{SD} 0.086)$ & $0.591(\mathrm{SD} 0.108)$ & 0.053 & 0.045 (0.0006 to 0.091$)$ \\
\hline Mean weight gain $(\mathrm{kg})$ & $9.35(\mathrm{SD} 2.58)$ & $9.8(\mathrm{SD} 3.11)$ & 0.49 & $-0.45(-1.78$ to 0.86$)$ \\
\hline Mean body fat accumulation ( $\%$ ) & $7.15(\mathrm{SD} 1.91)$ & $7.61(\mathrm{SD} 2.93)$ & 0.47 & $-0.45(-1.05$ to 1.40$)$ \\
\hline Mean BMD loss $\left(\mathrm{g} / \mathrm{cm}^{2}\right)$ & $0.052(\mathrm{SD} 0.024)$ & $0.037(\mathrm{SD} 0.034)$ & 0.037 & 0.015 (0.0009 to 0.029$)$ \\
\hline
\end{tabular}

$B M I$ body mass index, $S D$ standard deviation, $N S$ no significance, $M D$ mean difference, $C I$ confidence interval

A logistic regression analysis model was then constructed using the presence/absence of BMD loss as the dependent variable for the entire cohort. The probable significant parameters suspected clinically or identified in the previous analysis were then entered into the analysis in a stepwise fashion. The significant factors in the final equation included only early pregnancy BMD and fat accumulation in pregnancy, while the presence of GH was no longer a significant parameter. Specifically, a higher early pregnancy BMD was associated with higher BMD loss, with an odds ratio (OR) of 19 (confidence interval 1.3-27), while a higher fat accumulation in pregnancy was associated with lower BMD loss (OR 0.83, CI 0.74-0.93) (Table 3).

The highest mean arterial pressure recorded in the antenatal period in each patient was stratified into ten intervals from 70 to $170 \mathrm{mmHg}$, and then recoded into a "hypertensive index". This was used as a continuous variable and entered into a multiple linear regression model together with other probable parameters, using the BMD change in pregnancy as the dependent variable. Only early pregnancy $\mathrm{BMD}$, early pregnancy fat percentage and fat accumulation 
Table 3 Logistic regression model evaluating the association of probably variables with presence or absence of bone loss from early to late pregnancy

$S E$ standard error, $O R$ odds ratio, CI confidence interval

\begin{tabular}{lclllll}
\hline Variable & $B$ & SE & Wald & $P$ value & OR & $95 \%$ CI \\
\hline Age & -0.046 & 0.034 & 1.764 & 0.18 & 0.95 & $0.89-1.02$ \\
Body mass index & -0.036 & 0.075 & 0.233 & 0.62 & 0.96 & $0.83-1.11$ \\
Early pregnancy fat (\%) & -0.385 & 0.043 & 0.797 & 0.37 & 0.96 & $0.88-1.04$ \\
Early pregnancy BMD & 2.961 & 1.353 & 4.784 & 0.028 & 19.3 & $1.36-27.4$ \\
Weight gain in pregnancy & -0.036 & 0.049 & 0.525 & 0.46 & 0.96 & $0.87-1.06$ \\
Fat gain in pregnancy & -0.182 & 0.058 & 9.693 & 0.01 & 0.83 & $0.74-0.93$ \\
Parity & 0.385 & 0.250 & 2.36 & 0.12 & 1.47 & $0.90-2.40$ \\
Gestational hypertension & 1.23 & 0.904 & 1.86 & 0.17 & 3.43 & $0.58-20.2$ \\
\hline
\end{tabular}

Table 4 Multiple linear regression model evaluating probable factors affecting BMD loss in al pregnancies

\begin{tabular}{lcrl}
\hline Variable & $\begin{array}{l}\text { Standardized } \\
\text { coefficient }\end{array}$ & $t$ & Significance \\
\hline Early pregnancy BMI & 0.87 & 1.136 & 0.25 \\
Early pregnancy BMD & 0.228 & 4.982 & 0.001 \\
Early pregnancy fat (\%) & -0.19 & -2.425 & 0.016 \\
Weight gain in pregnancy & -0.026 & -0.508 & 0.61 \\
Fat accumulation in pregnancy & -0.191 & -3.491 & 0.001 \\
Gestational hypertension index & 0.087 & 1.863 & 0.063 \\
Parity & -0.021 & -0.453 & 0.65
\end{tabular}

Gestational hypertension index derived from stratified recoding of highest mean arterial blood pressure recorded before delivery

in pregnancy remained as significant variables in the equation and the hypertensive index was excluded (Table 4).

\section{Discussion}

Our data showed an obvious increase in weight and body fat percentage, as well as a demonstrable progressive fall in BMD of the os calcis from early pregnancy to late pregnancy. Univariate analysis showed a higher BMD loss during pregnancy in the gestational hypertensive group, but the effects of presence/absence of hypertensive disorders could not longer be seen on multivariate regression analysis. On the other hand, anthropometric parameters including early pregnancy BMD values, and early pregnancy fat percentages and fat accumulation were found to be more important factors affecting the extent of BMD loss in pregnancy.

Both GH and pre-eclampsia increases with increasing BMI and this was also observable in our data. A large retrospective cohort over 20 years have shown that increasing BMI was associated with an increased incidence of preeclampsia as well as other antenatal complications, and that underweight women actually had better outcomes than those with normal BMI [8]. As significant differences in the body mass index and early pregnancy body fat composition were observed in our cohort between those who developed hypertensive disorders and those who remained normotensive, it could be postulated that these basic anthropometric parameters were more likely to be responsible for the differences in BMD loss observed in pregnancy, rather than the gestational hypertensive condition.

Direct comparison of BMD loss in pregnancy between normotensive women and those with hypertensive disorders has only been reported in a few studies in the literature. In a previous longitudinal study form our group using the same methodologies to study the general trends in os calcis BMD in pregnancy, we have a higher but non-significant BMD loss in those with $\mathrm{GH}$ as compared to nonhypertensive controls $\left(0.049\right.$ vs. $\left.0.039 \mathrm{~g} / \mathrm{cm}^{2}\right)$ [9]. An older study that utilized radiological assessment of the bone density in the second metacarpal of normal women and those with pregnancy induced hypertension using the microdensitometry method showed subtle changes in the bone size and densities at various gestations of the pregnancies, but were unable to show a clear-cut difference in the BMD between the tow groups [10]. A more recent study that utilized a similar quantitative ultrasound system also showed a greater, but non-statistically significant degree of BMD loss in pre-eclamptic women as compared to normal controls. The same study also measured the relationship between insulin-like-growth factor-1 and osteocalcin as markers of bone turnover, and was able to show an altered relationship between concentrations of these markers in pre-eclamptic and normal women [11]. Summing up the data from these studies, it can be seen that the actual differences in BMD loss between pre-eclamptic and normotensive pregnant women, if any, are likely to be marginal or hardly undetectable.

The relationship between maternal calcium metabolism in pregnancy and the development of pre-eclamptic disorders has been a controversial issue in the literature. It is well established that pre-eclampsia is characterized by endothelial dysfunction related to abnormal trophoblastic invasion, and a potentially harmful maternal response to a semi-allogenic fetus that activates inflammatory cells and oxidative stress pathways, and cytokine profiles [12]. There 
was also some preliminary data to show that altered proinflammatory cytokines could be associated with decreased bone mass, clinically detectable osteoporosis and greater fracture risks in elderly age groups [13]. Calcium supplements have been established as a promising agent for prophylaxis against pre-eclampsia. In a review of 12 randomized controlled trials, calcium supplementation resulted in a significant $52 \%$ reduction in the relative risk of preeclampsia, with a particularly higher effect in those with low calcium intake [14]. When women were screened in early pregnancy for calcium excretion using a $24 \mathrm{~h}$ urine sample, it was found that those with excessive calcium excretion might be at risk of $\mathrm{GH}$ and pre-eclampsia later on [15]. In addition, using standard DXA BMD assessment in a small cohort before pregnancy and in 2 weeks postpartum period coupled with measurement of calcium intake confirmed that pregnancy was associated with decreases in whole-body and regional BMD, but that calcium intake was not a significant predictor of the skeletal response to pregnancy in well-nourished women [16]. Based on these data, it could be postulated that those with lower calcium reserves in early pregnancy, and thus higher pre-eclampsia risks, would also have an altered BMD loss rate because of their pre-pregnancy or early pregnancy calcium status. However, whether low calcium intake before or during pregnancy could be directly translated into subnormal BMD values into early pregnancy remains to be verified.

There were several limitations that can be seen in our data. While the serial measurements of BMD using quantitative ultrasound is entirely free of radiological hazards, and thus the ideal method for use during pregnancy, such systems were known to have a relatively larger coefficient of variation of around $2-3 \%$, as compared to BMD measured using standard radiological methods such as dual energy X-ray absorptiometry, which gives a coefficient of variation of around $1-2 \%$ for either short-term or long-term precision [17]. Thus, it has been argued that findings reported that using these ultrasound methods could be chance findings due to the high system variability. However, previous studies and our own data have demonstrated that the findings using such quantitative ultrasound has been consistent and valid when used to assess general BMD changes in pregnancy $[3,4,9,11]$, or when applied to specific antenatal conditions [18] or coupled with the assay of biochemical markers of bone metabolism [19]. The magnitude of BMD loss in pregnancy has been estimated to be in the range of 5-6\%, and should thus exceed the expected precision error and the minimal significant measurable differences of these systems. In addition, the incidence of $\mathrm{GH} /$ pre-eclampsia in this cohort was in par with the general obstetric population incidence, the number of genuine preeclamptics in the cohort was small. It could thus be argued that this small number of severe cases could attenuate any observable impact of pre-eclampsia on BMD loss in pregnancy, and thus our negative conclusion. A larger cohort that included more severe pre-eclampsia patients would be needed to confirm our results.

We conclude that the differences in BMD changes in pregnancy between those with gestational hypertensive disorders and those who remain normotensive should be small or negligible. Basic anthropometric parameters such as obesity that predisposes to GH should be more significant factors.

Conflict of interest The authors declare that they have no conflict of interest.

Open Access This article is distributed under the terms of the Creative Commons Attribution Noncommercial License which permits any noncommercial use, distribution, and reproduction in any medium, provided the original author(s) and source are credited.

\section{References}

1. Kaur M, Pearson D, Godber I, Lawson N, Baker P, Hosking D (2003) Longitudinal changes in bone mineral density during normal pregnancy. Bone 32:449-454

2. Wisser J, Florio I, Neff M, Konig V, Huch R, Huch A, von Mandach U (2005) Changes in bone mineral density and metabolism in pregnancy. Acta Obstet Gynecol Scand 84:349-354

3. Aguado F, Revilla M, Hernandez ER, Memdendex M, CortesPrieto J, Villa LF, Rico H (1998) Ultrasonographic bone velocity in pregnancy: a longitudinal study. Am J Obstet Gynecol 178:1016-1021

4. Pluskiewicz W, Drozdzowska B, Stolecki M (2004) Quantitative ultrasound at the hand phalanges in pregnancy: a longitudinal study. Ultrasound Med Biol 30:1373-1378

5. Guideline on Antenatal Care Part II (2008) Hong Kong College of Obstetricians and Gynaecologists, Sept 2008

6. The Management of Severe Pre-eclampsia/Eclampsia (2006) Green Top Guidelines. RCOG Guideline No 10 (A) March

7. Lowe SA, Brown MA, Dekker GA, Gatt S, McLintock CK, McMahon LP, Mangos G, Muller P, Paech M, Walters B (2009) Society of Obstetric Medicine of Australia, New Zealand guidelines for the management of hypertensive disorders of pregnancy 2008. Aust NZ J Obstet Gynaecol 49:242-246

8. Bhattacharya S, Campbell DM, Liston WA, Bhattacharya S (2007) Effect of body mass index on pregnancy outcomes in nullipaorous women delivering singleton babies. BMC Public Health 7:168

9. To WWK, Wong MWN, Leung TW (2003) Relationship between bone mineral density changes in pregnancy and maternal and pregnancy characteristics: a longitudinal study. Acta Obstet Gynecol Scand 82:820-827

10. Morikawa H, Chough SY, Ohara N, Yamasaki M, Ueda Y, Deguchi M, Mochizuki M (1989) Pregnancy induced hypertension (PIH) and osteoporosis. Nippon Naibunpi Gakkai Zasshi 65:1123-1134

11. Sowers M, Scholl T, Grewal J, Chen X, Jannausch M (2001) IGF-1, osteocalcin and bone change in pregnant normotensive and pre-eclamptic women. J Clin Endocrinol Metab 86:5898-5903

12. Yang GU, Lewis DF, Deere K, Groome LJ, Wang Y (2008) Elevated maternal IL-16 levels, enhanced IL-16 expressions in endothelium and leukocytes, and increased IL-16 production by placental trophoblasts in women with preeclampsia. J Immunol $181: 4418-4422$ 
13. McLean RR (2009) Proinflammatory cytokines and osteoporosis. Curr Osteoporos Rep 7:134-139

14. Hofmeyr GJ, Duley L, Atallah A (2007) Dietary calcium supplementation for prevention of preeclampsia and related problems: a systematic review and commentary. $\mathrm{Br} \mathrm{J}$ Obstet Gynaecol 114:933-943

15. Nielsen TR, Rylander R (2010) Urinary calcium and magnesium excretion relates to increase in blood pressure during pregnancy. Arch Gynecol Obstet Feb [Epub ahead of print]

16. Olausson H, Laskey MA, Goldberg GR, Prentice A (2008) Changes on bone mineral status and bone size during pregnancy and the influence of body weight and calcium intake. Am J Clin Nutr 88:1032-1039
17. Patel R, Blake GM, Rymer J, Fogelman I (2000) Long term precision of DXA scanning assessed over seven years in forty postmenopausal women. Osteoporos Int 11:68-75

18. To WWK, Wong MWN (2008) Bone mineral density changes in gestational diabetic pregnancies-a longitudinal study using quantitative ultrasound measurements of the os calcis. Gynecol Endocrinol 24:519-525

19. Tranquilli AL, Giannubilo SR, Corradetti A (2004) Ultrasound measurement of pregnancy induced changes in maternal bone mass: longitudinal cross-sectional and biochemical study. Gynecol Endocrinol 18:258-262 